\title{
23 Religious Diversity and Social Cohesion in German Classrooms: A Micro-Macro Study Based on Empirical Simulations
}

\begin{abstract}
The micro-macro transition is a core problem of sociological theory building. Micro-intentions and micro-behavior do not straightforwardly translate into corresponding phenomena on the macro level, due to potentially existing rival mechanisms and the dynamics and complexity of social interactions. This chapter proposes an integrated statistical approach to studying the micro-macro transition by combining a random coefficient multilevel approach with the Stochastic ActorOriented Model. This is elaborated for the substantively interesting and topical question whether the growing ethnic and religious diversity in our societies, along with the well-known tendency for homophily, necessarily lead to a decline in social cohesion. The German part of the CILS4EU data is used to tackle this empirically. We investigate how religious homophily plays out differently depending on the context defined by the composition of the classroom, and explore the potential of simulation methods to explain this macro-level phenomenon from micro-level network dynamics. The empirical puzzle as stated is answered by a model representing homophily in a straightforward way, taking account of the variability between classrooms and the uncertainty about the parameter values; but a closer analysis reveals a further puzzle, which we leave for future research.
\end{abstract}

\subsection{Introduction}

The growing diversity of Western societies, especially in ethnic and religious terms, has become a topic of major interest in social research. The seminal paper of Putnam (2007) has been especially influential, arguing that diversity is challenging the cohesion of modern societies. This hypothesis has stimulated an enormous, rapidly growing number of empirical papers speaking for or against this general claim (van der Meer and Tolsma 2014). The vast majority of the underlying analyses rely on standard

\footnotetext{
Note: We are grateful to Sebastian Pink and David Kretschmer for their work on the data set, and to them and also Christian Steglich for discussions and comments. We also thank a reviewer for helpful comments on an earlier version.
}

Tom A.B. Snijders, University of Groningen; University of Oxford Frank Kalter, University of Mannheim 
survey data, i.e., data sets with respondents as individual cases. As a rule, an individual-level variable, such as generalized trust, support for the welfare state, civic engagement, etc., is chosen as an indicator for social cohesion, and some variant of the regression approach is applied to estimate whether diversity as a context-level variable, often measured by the Herfindahl index, has an effect on the individual outcome variable controlling for other independent variables.

This research paradigm has certainly led to many helpful insights, the most obvious being that results are mixed, thus showing that the general claim is conditional and that careful differentiations are needed. Nevertheless, the dominance of the survey-based regression approach is somewhat surprising: social cohesion is a macro-level phenomenon, and applying regressions in the sketched way implicitly assumes that the macro phenomenon can simply be derived as a statistical aggregate of the individual outcomes. However, it has long been emphasized that the micro-macro transition is a core challenge in sociological theory building, and is often far from trivial. The keyword in this context is 'emergence'. Micro-intentions and micro-behaviors do not straightforwardly translate to phenomena at the macrolevel, due to the dynamics and complexity of social interactions. This has been widely stressed by sociologists (among many others, Raub 1984; Hedström and Swedberg 1998; Raub et al. 2011; Kalter and Kroneberg 2014).

Two classes of tools are especially suited to express social dynamics in the empirical micro-macro transition. On the one hand, network-analytical tools explicitly represent the dynamics of social interactions (e.g., Snijders 2001; Stokman and Vieth 2004). Macro-level characteristics of social networks can directly capture the idea of social cohesion (Moody 2001; Kalter 2016; Kalter and Kruse 2014). Network analysis allows to model diverse mechanisms of social interaction producing macro-level phenomena, and to test the validity of these mechanisms empirically. Next to this, agentbased modelling has become a major workhorse in dealing with phenomena of emergence. An agent-based model (ABM) is a model of individual actors interacting with each other and with environmental constraints over time (Epstein 2006). ABMs are programmed in computer language and analysed inductively: by iterating the assumed agent behaviour in the context of many other agents dynamically over time, ABMs allow to investigate the macro-level consequences of this interaction (Macy and Willer 2002; Manzo 2010).

Stochastic actor-oriented models (SAOM) for network dynamics (Snijders 2001; Steglich et al. 2010; Snijders and Steglich 2015) combine both of these tools. Basically, they are agent-based models that make assumptions about the behavior of actors, foremost their choices of building ties to other actors. In addition, they incorporate elements of generalized linear statistical models and confront the assumptions with empirical data. Correspondence with observed network-level descriptives allows to assess the goodness of fit of model assumptions, and to estimate parameters determining the behavior of actors from empirical data. 
In this paper we use the SAOM as implemented in the SIENA software (Ripley et al. 2020) in an integrated empirical approach to the micro-macro transition. We study the impact of religious diversity on social integration in classrooms of adolescents, relying on the network data contained in the first two waves of the German part of the CILS4EU data (Kalter et al. 2013). We describe the general pattern of the relation between religious diversity and social cohesion and then employ simulation methods to figure out in how far they may be explained by various network formation mechanisms.

\subsection{Theory and past research}

'Social cohesion' is a key term in the social sciences, and as is the case for many key terms, it has been used in inconsistent and often vague ways. In empirical research it has been operationalized by trust, civic engagement, attitudes towards the welfare state, and many other concepts (van der Meer and Tolsma 2014; Schaeffer 2013). In a straightforward understanding, however, it refers to the social ties between the members of a community or society, and network analysis provides a lot of measures to give it a precise meaning (e.g., Scott and Carrington 2011). Whatever the precise measure, this view emphasizes that social cohesion is a macro-level result of individual-level processes of tie formation.

When asking why diversity in general, and religious diversity specifically, could have an impact on social cohesion in these terms, the most obvious reason certainly is homophily. One of the most robust findings in the social sciences is that people tend to have more ties to others who are similar to themselves (McPherson et al. 2001), and religious homophily is a well-known manifestation (e.g., Windzio and Wingens 2014; Cook et al. 2017). While the tendency to relate to similar others is partly a matter of the opportunity structure (Blau 1977), which has also been called 'baseline homophily' (McPherson et al. 2001, p. 419), empirical network analysis has shown that this tendency is strong also net of the mere availability of contacts. This is sometimes referred to as 'inbreeding homophily' and holds for quite a range of characteristics, e.g., age, sex, occupation, education, ethnicity (Moody 2001; Mouw and Entwisle 2006; Wimmer and Lewis 2010), and in the USA it has proved to hold also, and especially strongly, for religion (Cheadle and Schwadel 2012).

The mechanisms behind 'inbreeding homophily' are less clear and it is challenging to disentangle them in empirical analyses. The most obvious starting point to explain homophily is to trace them back to individual preferences.

Sharing characteristics reduces the cognitive and physical costs of communicating, anticipating and evaluating behavior, building mutual expectations, developing trust, etc. (Kossinets and Watts 2009). There is also support for the idea that similar others are found to be more attractive in appearance (e.g., Byrne 1971; 
Huston and Levinger 1978). While religion is not a visible characteristic per se, wearing religious symbols - such as cross necklaces or headscarves - might be signals in the process leading to the creation of ties; most importantly, however, sharing religious world views seems, like psychological factors in general, particularly relevant for the costs and utilities of maintaining already existing ties (Felmlee et al. 1990). Religions may even contain explicit norms to prefer fellow believers.

Note that religion, in given contexts, is usually empirically correlated with other characteristics that may also foster a preference for similar people, most importantly ethnicity which also encompasses linguistic and other cultural elements, but also socio-economic class. Thus, preferences for social status, the same language, or other cultural aspects in the choice of social ties, can lead to religious homogeneity, and it will be empirically challenging to disentangle the true reasons (McPherson et al. 2001; Moody 2001). Note also that even within a given clear-defined opportunity structure, such as a classroom, the choice of friends may depend on reasons that are related to other, unrelated opportunity structures. Most obviously, in our case, students within the same classroom might also meet in their leisure time in religious places, like churches or mosques, and their friendship might predominantly arise from the time spent around these events. In contrast to 'availability effects' that arise from the opportunities in the context under investigation (i.e., the classroom), these kinds of additional opportunities arising in further organizational contexts have been called 'propinquity' effects (Wimmer and Lewis 2010).

Whatever the more detailed mechanism behind religious homophily, its existence would suggest that - net of additional mechanisms and in a straightforward aggregation of individual choices - social cohesion, vaguely defined for the moment, would decrease with religious diversity. Higher diversity, by definition, means a lower likelihood that two randomly chosen individuals share a characteristic; therefore, if this characteristic is associated with homophilous preferences, higher diversity is associated with a lower likelihood that they form a social tie.

However, the pattern of ties is not a mere aggregation of individual homophilous preferences, and there are a series of additional mechanisms determining tie formation. Some of these mechanisms might amplify the effects of homophily, others might counteract. The most prominent, and empirically most firmly established of these mechanisms are reciprocity (an early reference, in a German school context, is Delitsch 1900) and transitive closure (e.g., Davis 1970). Reciprocity means that the likelihood to choose someone as a friend is increased, if this person in turn has chosen oneself as a friend. To understand the deeper mechanism behind this tendency, Social Exchange Theory (Emerson 1976) is a fruitful starting point (also see Block 2015). Friendship is basically regarded as an investment, and mutuality helps to increase the expected rewards in relation to the costs. Transitivity denotes, loosely speaking, the phenomenon that a person is more likely to choose another as a friend, if there is a common third friend. Here standard explanations build on opportunity structure arguments, following classical ideas of Simmel (1950) or Granovetter (1973), on the one 
hand, and on the more attitudinal mechanisms of classical Balance Theory (Heider 1948), on the other hand (again, see Block 2015). A first major attempt to figure out empirically how these more general network formation mechanisms influence the macro-micro-macro relation between diversity and social cohesion is formed by the analyses of Kalter and Kruse (2014). They use the first wave of the CILS4EU data, for all included countries. They study the consequences of ethnic diversity, as expressed by the Herfindahl index, on social cohesion measured in several ways: the density of the friendship network, the reachability (defined as average reciprocal geodesic distance) in the friendship network, and estimated coefficients for Exponential Random Graph Models. Basically, they find that, despite a clear and strong tendency for ethnic homophily, there is hardly any relation between ethnic diversity and social cohesion.

\subsection{Data}

We study the consequences of religious heterogeneity on social cohesion, using longitudinal network data from the German part of the Children of Immigrants Longitudinal Study in Four European Countries (CILS4EU) (Kalter et al. 2017). This comparative panel study was started in late 2010, interviewing adolescents that were about 14 years of age in wave 1. In the first step of the sampling process, schools were drawn from a nationwide list of schools that enroll students at this age; depending on the expected share of children of immigrants, schools were classified into four different strata and disproportionate stratified random sampling was applied, oversampling schools with higher proportions. In the second step, as a rule, two classrooms were randomly chosen within each school. In the third step, all students within this classroom were selected. The German sample of the wave-1 data comprises 144 schools, 271 classrooms and 5,013 students (Kruse and Jacob 2016). A sociometric module could successfully be administered in 267 of these German classrooms. It contains, among others, the nomination of the up to five best friends within the classroom. In wave 2, a year later, the same module could be applied again in 203 of these classrooms (Kruse et al. 2016). Our analysis is based on waves 1 and 2 . We selected all classrooms where at least 10 students participated in wave 1 , and likewise for wave 2 . These were 140 classrooms.

\subsection{Classroom cohesion}

In our micro-macro study, the micro level is defined by the individual student, and the macro level by the classroom. We consider diversity with respect to religion, focusing on the main minority religion in Germany, Islam, and following a binary approach where the minority is defined by the Muslim students and the majority by all nonMuslim students. (Thus, we are imputing a common religion to all non-Muslims ....) 
We study how religious heterogeneity of the classroom affects social cohesion. The heterogeneity is reflected by the proportion of Muslim students in the classroom, denoted by $p$.

As mentioned above, network theory provides a rich variety of measures for social cohesion differentially emphasizing its various sub-aspects. Moody and White (2003) give a very sophisticated treatment. For the purposes of this paper we decided to work with a straightforward structural measure that is simple and easy to interpret. It is based on the reciprocated degree, defined as the number of reciprocated friendships of an individual. Because of our focus on majority and minority processes, we employ two measures of social cohesion in a classroom: the classroom average of the reciprocated degrees of its majority (non-Muslim) students, and the same average for its minority (Muslim) students. This ranges in the data from 0 to 5 , and the majority as well as the minority have a mean of 2.3. This pays no attention to issues of integration and connectedness, but may be considered a basic measure that may be considered in future work together with other measures of structural cohesion such as studied in Moody and White (2003) and Kalter and Kruse (2014).

Figure 23.1 shows the scatter plot of the average number of reciprocal friendships per classroom in wave 2, for each religious category separately, in dependence on the proportion $p$ of Muslims in the classroom, with a smooth approximation. The plot shows decreasing curves, not far from linear, for $p$ up to 0.5. Perhaps they are increasing for greater proportions; but the number of classrooms there is low, and it is not clear from the plot whether this conclusion is warranted.

To further assess this relationship we conducted a bivariate regression with classrooms as cases, and as the two dependent variables the average reciprocated degrees for the two religious categories in wave 2 . The independent variables were $p$ and, as a control variable, the total number of students used in the analysis for this classroom - this was the maximum possible number of reciprocated friendships; its mean is 20.9. The analysis was done for the 111 classrooms with $p<0.5$ and having at least 10 respondents in both waves. The plot shows that in this range, the effect of $p$ is approximately linear. The regression coefficients did not differ significantly between the two dependent variables. The bivariate regression was calculated using function gls in package nlme of the statistical system R.

Table 23.1 shows that there clearly is a decreasing effect of $p$ in the range $0 \leq p<0.5$ (the interaction between $p$ and religion was not significant). The expected average number of reciprocal ties in a classroom without any Muslims $(p=0)$, and an average size of 20.9, is equal to 2.1. For $p=0.5$ this drops to 1.6 (and 1.5 for the Muslims in such a class). The drop from 2.1 to 1.6 is considerable. 


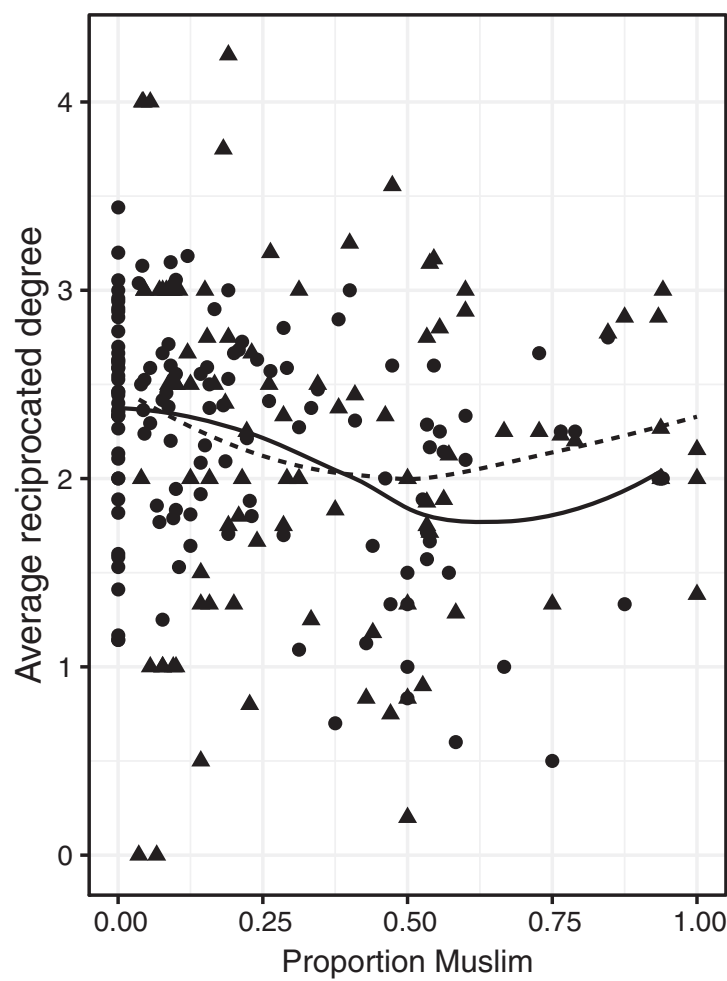

\section{Religion}

$\rightarrow$ Majority

- A. Minority

Figure 23.1: Average number of friendship nominations per classroom in wave 2, as a function of the proportion of Muslim students in the classroom, for majority and minority students.

Table 23.1: Bivariate regression of the average reciprocated degree, for minority and majority students, on the proportion minority students; $N$ (classrooms) $=111$.

\begin{tabular}{lrc}
\hline Effect & par. & (s.e.) \\
\hline Fixed part & & \\
Intercept & 1.796 & $(0.251)$ \\
Religion & -0.099 & $(0.114)$ \\
Proportion minority $(p)$ & -0.961 & $(0.386)$ \\
Number of students with available data & 0.015 & $(0.011)$ \\
\hline Random part & & \\
Variance majority & 0.330 & \\
Variance minority & 0.603 & \\
Correlation & 0.418 & \\
\hline
\end{tabular}




\subsection{The empirical puzzle}

Given that there is homophily to some extent with two groups, one being the majority and the other the minority, a straightforward expectation about the proportions in which the network is divided would be that, when the proportion of one group becomes larger, the average number of friends will become larger for this group and smaller for the other group, as a consequence of the availability of potential friends in the own group. This means that qualitatively and in a first approximation, we understand that in Figure 23.1 the curve for the majority is declining for minority proportions from 0 to 0.5 , but not that for the minority, Muslims, it also is declining.

The empirical question therefore is twofold.

1. Why is the average number of within-classroom reciprocated friendships for nonMuslim students declining as a function of the proportion of Muslims, for proportions less than 0.5? Can we understand the numerical value of this decline?

2. Why is the average number of within-classroom reciprocated friendships for Muslim students declining as a function of the proportion of Muslims, for proportions less than 0.5? Can we understand the numerical value of this decline?

For the students in the majority group, our intuitive reasoning already seems to provide an answer to the first question; but we would like to back up this intuition with a formal empirical model, and study numerically the size of this decline. This is done in the next section. For this we use the Stochastic Actor-oriented Model, a model making the macro-micro-macro connection explicit. This will also serve as the starting point for studying the second question.

To express our two questions, we use two coefficients: the regression coefficient of the average reciprocated degree on $p$, controlling for the number of participating students in the classroom, where averaging is across the majority students; and this regression coefficient where averaging is across the minority students. We used only the 111 classrooms with at least 10 respondents in wave 1 and also in wave 2, and with less than 50\% Muslim students, to avoid ambiguity about the definition of the minority. For this set of classrooms, the regression coefficient for the minority students is -1.23 ; for the majority it is -0.94 . The question now is whether we can find satisfactory micro-level models to explain these numbers.

\subsection{Multilevel network analysis}

A model that represents the dynamics of network choices by students, given the context composed of the classroom, the attributes of all its students, and the current state of the network, is the Stochastic Actor-oriented Model ('SAOM'), proposed in Snijders (2001) and further explained in Snijders et al. (2010) and Snijders (2017). 
It is implemented in the RSiena package (Ripley et al. 2020). The 'macro' here is the small environment consisting of the classroom, the 'micro' is the student who makes friendship choices. In the SAOM as applied to this case the set of actors is composed of the students in one classroom and the network is the structure of all friendship ties between them. It is assumed that the actors have control over their friendship choices, i.e., their outgoing friendship ties. The model takes the first observation of the network (wave 1) as given, and the dependent variable is the network at the second observation, i.e., wave 2 . It assumes that the change from one network observation to the next is the result of a large number of sequential small changes, so-called microsteps. In a micro-step one of the actors makes a choice in which the options are to create one new friendship tie, to withdraw one existing friendship tie, or to leave the network unchanged. The 'current network' changes gradually as a result of the microsteps, from the first observed network to the second observed network. The choice in the micro-step is made with probabilities according to a generalized linear model in which the explanatory variables, called 'effects', are functions of the current network structure and the attributes of the actors. These probabilities can be derived from a myopic stochastic optimization principle, in which each actor optimizes a linear combination of the effects, called the 'evaluation function', to which random disturbances are added; the optimization considers only the direct result of this choice, without further strategic considerations. Detailed specifications are in Snijders et al. (2010). The choice of the effects, just like any model statistical specification, depends on theoretical considerations and empirical fit. The SAOM is applied here to the network dynamics from wave 1 to wave 2 of the CILS4EU data.

Figure 23.2 is Coleman's diagram (Coleman 1990; Raub et al. 2011) for the case of our study: on the basis of the classroom composition we wish to explain social cohesion as measured by the average reciprocated degree.

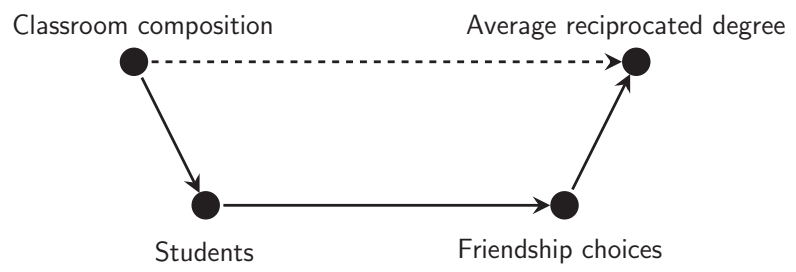

Figure 23.2: Coleman's scheme for this study.

The stochastic agent-based model at the core of the SAOM (Snijders et al. 2010; Snijders and Steglich 2015) is the basis of our macro-micro-macro approach. The bridge assumptions are represented by the specification of the SAOM, and how this depends on ethnicity and classroom composition; the assumptions about individual behavior are the probabilities of tie changes in the SAOM, which can be summarized 
as myopic context-dependent goal-oriented behavior; and the transformation rule is the sequence of small changes (micro-steps) that takes the network from one observed wave to the next observed wave, each change also implying a change in the network context for all actors. The macro-level measure is a simple average of the actor-level reciprocated degrees, but it is only the end point of this quite complex transformation rule, and not a direct aggregate of individual choices.

We have quite a large set of German classrooms in the CILS4EU data, giving us ample variation in macro-level conditions. To handle the large number of classrooms, we need a multilevel version of the SAOM. Multilevel network analysis is discussed in Snijders (2016, p. 31-36). A multilevel SAOM is a combination of SAOMs, one for each classroom, all with the same model specification, but with possibly different parameters. To define the multilevel SAOM we have to specify how the parameters of the various classrooms are related, and how they are estimated. The simplest specification is the multi-group option (Ripley et al. 2020, Section 11.1), which assumes that all groups have the same parameter value. Even though this is quite a drastic assumption, it provides an illuminating first step in our micro-macro approach. A more reasonable approach is to assume that the parameters of the groups vary freely, and are estimated for each group separately. This approach resembles meta-analysis (Snijders and Baerveldt 2003). It was applied, e.g., in Knecht et al. (2010), where this approach posed some computational problems, because many of the school classes were too small to allow sound estimation by the methods implemented in RSiena; this was managed by a drastic reduction in the number of usable classrooms. The second step in this chapter follows a different approach: the integrated hierarchical multilevel approach developed in Koskinen and Snijders (2020), of which the implementation is described in Ripley et al. (2020, Section 11.3), as explained below.

\subsubsection{First step: Multi-group approach}

As a first step we try to answer our two questions while postulating that in each classroom the friendship network develops according to a Stochastic Actor-oriented Model with identical parameters across classrooms, but taking into account the different classroom compositions. As discussed above, we expect intuitively that the average reciprocated degree for students from the majority declines as a function of $p$, and increases for the minority. Therefore we do not expect that this initial model will provide the answer to both of our questions; but it is a check on the correspondence between our intuitive arguments and the Stochastic Actor-oriented Model.

The model specification was chosen parsimoniously and according to the current best practice. The effects included are explained in Snijders et al. (2010) and Ripley et al. (2020). The outdegree effect is like an intercept in other statistical models, and represents the balance between creating and dropping ties. Given that the 
network is sparse, so potentially many more ties can be created than can be dropped, it usually has a negative parameter. Reciprocity and transitivity are basic features of network dynamics, represented by the reciprocity and transitive triplets effects. The 'transitive reciprocated triplets' is an interaction between reciprocity and transitivity, expected to be negative (Block 2015). Differential centrality of nodes is represented by the indegree-popularity, outdegree-activity, and outdegree-popularity effects; these reflect, respectively, variance of indegrees, variance of outdegrees, and the covariance between these. The 'reciprocal degree-activity' is the effect of the focal actor's reciprocated degree on tie creation and maintenance. This represents a 'saturation effect': an actor with a higher reciprocated degree is expected to have less value for additional ties, so that the parameter for this effect is expected to be negative. Sex homophily is usual in secondary school friendship networks, and represented by the 'different sex' effect. For religion, used here as a binary variable, the three basic effects are included, viz., the 'different religion' effect, the religion of the receiver (alter), and the religion of the sender (ego) of the friendship tie. The 'different religion' effect is expected to be negative, which represents the basic assumption in our micro-macro model, that being of a different religion has a negative effect on friendship creation and maintenance.

For the multi-group analysis, to achieve good convergence of the estimation algorithm, we included classrooms according to a somewhat more stringent criterion than above. To the requirements that both waves should have at least 10 respondents and the proportion Muslims should be less than 0.5, we added the criterion that there are at least 12 students for whom the reciprocal degree in wave 2 is nonmissing, and that these are more than $60 \%$ of the total number in their classroom. This left 101 classrooms from the 111 selected in the previous section. For this data set, the regression coefficient of the average reciprocal degree on the proportion of Muslim students in the classroom is -1.16 for the minority students, and -0.74 for the majority.

Estimating this model under the assumption of equal parameters across groups led to the parameter estimates in Table 23.2. The estimated parameter values are in line with what is usually found for friendship networks in secondary schools. In particular, we see clear evidence for homophily with respect to sex and religion.

To see the implications of this model for the macro level, we simulated 1,000 data sets for the combined 101 schools according to the model of Table 23.2. For each simulated data set we computed the two regression coefficients of interest, i.e., the effect of the proportion of Muslim students on the average reciprocated degree for minority and majority students separately. This procedure is similar to the goodness-of-fit procedure usual for SAOMs (Lospinoso and Snijders 2019), but now applied to the multi-group situation. Figure 23.3 shows the distributions of these regression coefficients in violin plots.

For the minority students most of the distribution is in the positive range, for the majority students most is in the negative range. This is in correspondence to 
Table 23.2: Parameter estimates and standard errors for the multi-group estimation of the Stochastic Actor-oriented Model, $N=101$ schools.

\begin{tabular}{lrr}
\hline Effect & estimate & (s.e.) \\
\hline outdegree (density) & -0.809 & $(0.150)$ \\
reciprocity & 2.902 & $(0.175)$ \\
transitive triplets & 0.510 & $(0.028)$ \\
transitive reciprocated triplets & -0.178 & $(0.045)$ \\
indegree - popularity & -0.005 & $(0.018)$ \\
outdegree - popularity & -0.019 & $(0.053)$ \\
outdegree - activity & -0.114 & $(0.020)$ \\
reciprocated degree - activity & -0.231 & $(0.043)$ \\
different sex & -0.236 & $(0.034)$ \\
different religion & -0.177 & $(0.044)$ \\
religion Muslim alter & -0.031 & $(0.048)$ \\
religion Muslim ego & -0.084 & $(0.051)$ \\
\hline
\end{tabular}

Notes: Convergence $t$ ratios all $<0.06$.

Overall maximum convergence ratio 0.14 .

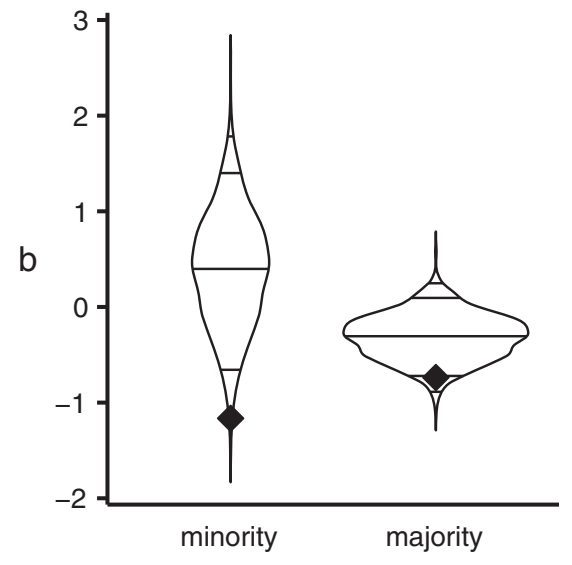

Figure 23.3: Distributions of regression coefficients of average reciprocated degrees on $p$ for multigroup model. Horizontal lines denote quantiles at 0.01, 0.05, 0.50, 0.95, and 0.99; diamonds represent observed values.

our intuitive ideas that the availability of more potential friends in the own group will result in more reciprocated friendships; although the probabilities of these expected patterns are not very high. But we also see that the observed values are situated very low in the distributions; for the majority it is at percentile 0.10 , for the minority at 0.01 . The correspondence between the model predictions and the observed regression coefficient for the majority is a confirmation of our intuitive reasoning. However, this multi-group model does not correspond satisfactorily to our data with respect to the minority. Concluding, this model is sufficient to answer our first empirical question, but not the second. 


\subsubsection{Second step: Integrated multilevel approach}

Could our questions then be answered, still by assuming that the model specification is identical between the classrooms, but the parameter values vary? This is more realistic than the previous approach. This is not expected to give systematically different average values for our regression coefficients, but there may be more random variability in a realistic way, which could imply that the data are not really as extreme as they seem to be in Figure 23.3.

The random coefficient multilevel version of the SAOM is developed in Koskinen and Snijders (2020). It extends the multilevel approach of Snijders and Baerveldt (2003) by assuming that the network in each classroom evolves according to a SAOM with the same specification, but different parameter vectors, these classroom-level parameter vectors having a multivariate normal distribution. This is similar to the Hierarchical Linear Model of multilevel analysis (Snijders and Bosker 2012), but now for longitudinal network data. A Bayesian estimation procedure for this model is implemented in the function sienaBayes of the R package RSienaTest (Ripley et al. 2020).

Convergence of the estimation posed some problems, and it was necessary to drop one classroom, because in attempts to estimate the model for the set of 101 classrooms it was an outlier, differing too strongly from the other classrooms. On inspection this classroom appeared to be composed of only majority students, with the highest average degree of all classrooms, 4.5. The regression coefficient of the average reciprocal degree on the proportion of Muslim students in the remaining set of 100 classrooms for the majority is -0.73 . The conclusion for the multi-group approach for this set of 100 classrooms is the same as for the 101 classrooms analyzed above.

All SAOM parameters were assumed to vary randomly between classrooms. The macro-level parameters of the multilevel SAOM are the expected values and the between-classroom variances of the classroom-level parameters. Their estimates are presented in Table 23.3. Most of the estimates are similar to those in Table 23.2; the effects that are significant in both models, which are most, have the same sign. To the extent that there are differences, clearly the results from Table 23.3 are more credible, being based on more plausible model assumptions.

For this model, since it is Bayesian, we can use the so-called posterior predictive distribution (see, e.g., Jackman 2009) to check the implications and the model fit. In this case, this is the distribution where the set of 101 schools is fixed, as well as the composition of the classrooms and the friendship networks at wave 1 , but the probability distributions are random: 'a sample from what the SAOM parameters possibly could have been'; and the networks at wave 2 also are random: 'a sample of what could have occurred in these schools given the sampled parameters'. The posterior predictive distribution reflect this double stochasticity. The posterior predictive distribution of our two regression coefficients, for the model of Table 23.3, are presented in Figure 23.4 again by violin plots. 
Table 23.3: Posterior means and standard deviations for multilevel SAOM analysis for $N=100$ classrooms.

\begin{tabular}{lrrr}
\hline Effect & par. & (psd) & betw. sd \\
\hline outdegree (density) & -1.336 & $(0.105)$ & 0.432 \\
Reciprocity & 2.338 & $(0.095)$ & 0.278 \\
transitive triplets & 0.546 & $(0.025)$ & 0.121 \\
transitive reciprocated triplets & -0.199 & $(0.035)$ & 0.158 \\
indegree - popularity & 0.044 & $(0.013)$ & 0.087 \\
outdegree - popularity & -0.118 & $(0.025)$ & 0.130 \\
outdegree - activity & -0.099 & $(0.017)$ & 0.076 \\
reciprocated degree - activity & -0.100 & $(0.026)$ & 0.101 \\
different sex & -0.320 & $(0.038)$ & 0.205 \\
different religion & -0.227 & $(0.064)$ & 0.250 \\
religion Muslim alter & 0.038 & $(0.058)$ & 0.212 \\
religion Muslim ego & 0.092 & $(0.072)$ & 0.286 \\
\hline
\end{tabular}

Notes: par = posterior mean; psd = posterior standard deviation;

betw. $s d=$ posterior between-groups stand. deviation.

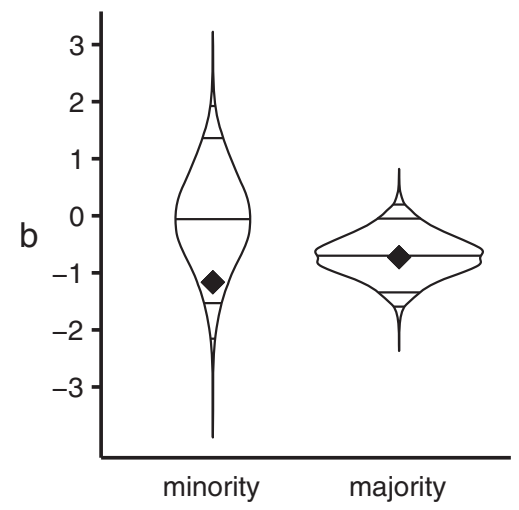

Figure 23.4: Distributions of regression coefficients of average reciprocated degrees on $p$ for random coefficient model. Horizontal lines denote quantiles at $0.01,0.05,0.50,0.95$, and 0.99 ; diamonds represent observed values.

As expected, the difference with Figure 23.3 is mainly that the distribution in Figure 23.4 is more spread out (note the scale difference on the vertical axis). For the majority students the observed value is quite in the middle of the distribution (at percentile 0.47 ), and for the minority students it is still on the low side but not exceptional (at percentile 0.08). This means that for the majority as well as for the minority students, in the light of the variability between classrooms and the regular friendship network processes, there is nothing surprising about the observed negative regression coefficients. We may consider that both our questions can be answered by the SAOM, assuming parameter values to vary between the classrooms, and given the uncertainty that we have about their distribution. 


\subsubsection{And yet ...}

Our empirical puzzle focused on the regression coefficients for the average reciprocated degree on the proportion of minority students, and we answered it by considering posterior predictive checks for our macro-micro-macro statistical model. It should be noted, however, that such model checks are not an overall test of goodness of fit for the model. Posterior checks for statistics that are chosen for their descriptive interest may be rather forgiving, because such statistics may include a lot of variability that is not of diagnostic interest for the model. For example, the regression coefficients used depend also on the standard deviations of the average reciprocated degrees.

Therefore, for the purpose of model checking, we also consider the fit for a statistic that borrows less variability from elements of the model that are not of primary interest. Such a statistic is the partial correlation between the average reciprocated degree and the minority proportion, controlling for the number of cases and for the average reciprocated degree in the first wave. This partial correlation in our data set is -0.55 for the minority students, and -0.03 for the majority students. Figure 23.5 shows that this value is far from being reproduced by the random coefficient model of Table 23.3 for the minority students, although it is totally in line with respect to the majority students. If we had formulated our empirical puzzle in terms of this partial correlation coefficient, this random coefficient model would not have solved it.

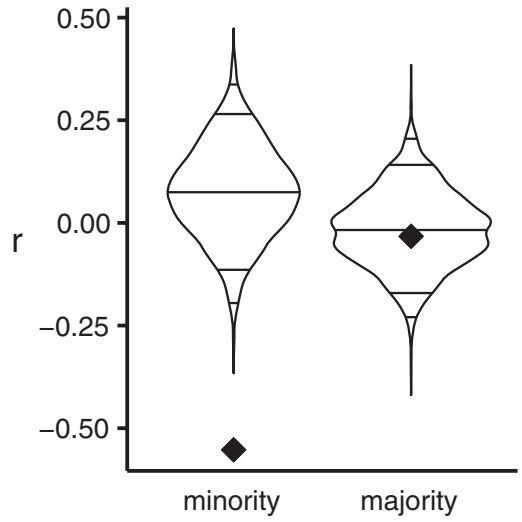

Figure 23.5: Distributions of partial correlations of average reciprocated degrees with $p$ for random coefficient model. Horizontal lines denote quantiles at 0.01, 0.05, 0.50, 0.95, and 0.99; diamonds represent observed values.

\subsection{Summary and conclusion}

In this chapter we have tried to contribute to studying a key sociological question with an explicit notion of the micro-macro challenges involved. The macro-level consisted of the classroom (still small for macro), and there were a large number of macro-level cases. The micro-level consisted of the students. The research was about the dynamics 
of friendship networks between the students measured at two waves, using the CILS4EU data. We have built on Knecht, Snijders, Baerveldt, Steglich and Raub (2010) where a similar multilevel network study was conducted, but without the explicit micro-macro focus; on Kalter and Kruse (2014) where a micro-macro study using the CILS4EU data was done, but without explicitly using network dynamics for the microlevel model; on Snijders and Steglich (2015), a micro-macro study using the SIENA framework, but not guided by a clear empirical puzzle; and on Koskinen and Snijders (2020), a multilevel network model.

Taking the relation between religious diversity and social cohesion as the substantive example, we used fundamental assumptions about network dynamics as the micro-level mechanisms, and tested to which extent these are sufficient to understand a particular macro-level characteristic. For the empirical test, we used rich multi-level longitudinal network data and recent elaborations of the Stochastic Actor-oriented Model. This enabled us to pursue an integrated approach to the macro-micro-macro question. An empirical puzzle, challenging common theoretical intuitions, served as the guideline in successively increasing the level of detail in the theoretical argumentation and the statistical implementation.

The puzzle consisted of two parts. We found that the regression coefficient of the classroom average reciprocated degree on the proportion of minority students is negative for the majority; and that it is also negative for the minority. Given a tendency to homophily, the first empirical result agreed with our intuitive expectations, and we expected no problems to confirm the empirical finding that this coefficient is negative. The second, however, was more puzzling. When the own group (minority) is larger and there is homophily, one would expect that the number of reciprocated friendships will be larger.

We approached these questions by applying a Stochastic Actor-oriented Model, specified in the usual way, assuming homophily. We focused not only on the signs, but wanted to find models giving a good correspondence with the values of the regression coefficients. When the assumption was that the parameters of the network model are identical across all classrooms, the regression coefficient found for the majority was well explained; but not so for the minority. However, after relaxing this auxiliary assumption by assuming that the classroom-level parameters are a sample from a multivariate normal distribution, also the coefficient for the minority was not unexpected at all; although, confirming our intuition, the estimated probability of a positive coefficient in our model was still larger than 0.5.

Therefore, one might say the puzzle is solved. However, the puzzle reappeared when we considered a less forgiving statistic: the partial correlation between average reciprocated degree and minority proportion, controlling for the average reciprocated degree at the earlier wave, is for the minority much lower than what could be reproduced by our Stochastic Actor-oriented Model with varying parameters.

Our puzzle was solved but the solution revealed a new puzzle, on which we hope to work in future. However, we did make substantive progress, and we hope 
to continue with further theoretical and statistical enrichments. We do not regard this paper as the last tale on the substantive issues. Rather we understand it as a demonstration of the general fruitfulness of this approach, which comprises the collection of rich and demanding data and sophisticated elaborations of statistical modelling to detect theoretical desiderata, and which we hope will improve our understanding of micro-macro phenomena in the social world.

\section{References}

Blau, Peter M. 1977. Inequality and Heterogeneity: A Primitive Theory of Social Structure. New York: Free Press.

Block, Per. 2015. "Reciprocity, transitivity, and the mysterious three-cycle." Social Networks 40: 163-173.

Byrne, Donn. 1971. The Attraction Paradigm. New York: Academic Press.

Cheadle, Jacob E. and Philip Schwadel. 2012. “The 'friendship dynamics of religion,' or the 'religious dynamics of friendship'? A social network analysis of adolescents who attend small schools." Social Science Research 41:1198-1212.

Coleman, James S. 1990. Foundations of Social Theory. Cambridge/London: Belknap Press of Harvard University Press.

Cook, J. Benjamin, Philip Schwadel, and Jacob E. Cheadle. 2017. "The origins of religious homophily in a medium and large school." Review of Religious Research 59(1):65-80.

Davis, James A. 1970. "Clustering and hierarchy in interpersonal relations: Testing two graph theoretical models on 742 sociomatrices." American Sociological Review 35:843-852.

Delitsch, Johannes. 1900. "Über Schülerfreundschaften in einer Volksschulklasse." Zeitschrift für Kinderforschung 5:150-163.

Emerson, Richard M. 1976. "Social exchange theory." Annual Review of Sociology 2:335-362.

Epstein, Joshua M. 2006. Generative Social Science: Studies in Agent-Based Computational Modeling. Princeton, NJ: Princeton University Press.

Felmlee, Diane, Susan Sprecher, and Edward Bassin. 1990. "The dissolution of intimate relationships: A hazard model." Social Psychology Quarterly 53:13-30.

Granovetter, Mark S. 1973. "The strength of weak ties." American Journal of Sociology 78: 1360-1380.

Hedström, Peter and Richard Swedberg (eds.). 1998. Social Mechanisms: An Analytical Approach to Social Theory. Cambridge: Cambridge University Press.

Heider, Fritz. 1948. "Attitudes and cognitive organization.” Journal of Psychology 21:107-112.

Huston, Ted L. and George Levinger. 1978. "Interpersonal attraction and relationships." Annual Review of Psychology 29:115-156.

Jackman, Simon. 2009. Bayesian Analysis for the Social Sciences. Chichester: Wiley.

Kalter, Frank. 2016. "Social network analysis in the study of ethnic inequalities." In Emerging Trends in the Social and Behavioral Sciences, eds. Robert A. Scott and Stephen M. Kosslyn. Oxford: Wiley, 1-15. doi:10.1002/9781118900772.etrds0397.

Kalter, Frank, Anthony F. Heath, Miles Hewstone, Jan O. Jonsson, Matthijs Kalmijn, Irena Kogan, and Frank van Tubergen. 2013. Children of Immigrants Longitudinal Survey in Four European Countries (CILS4EU): Motivation, Aims, and Design. Technical report, GESIS: GESIS Data Archive, Cologne. 
Kalter, Frank, Anthony F. Heath, Miles Hewstone, Jan O. Jonsson, Matthijs Kalmijn, Irena Kogan, and Frank van Tubergen. 2017. Children of Immigrants Longitudinal Survey in Four European Countries (CILS4EU) - Full Version. Technical report, GESIS: GESIS Data Archive, Cologne. ZA5353 Data file Version 3.3.0, doi:10.4232/cils4eu.5353.3.3.0.

Kalter, Frank and Clemens Kroneberg. 2014. "Between mechanism talk and mechanism cult: New emphases in explanatory sociology and empirical research." KZfSS Kölner Zeitschrift für Soziologie und Sozialpsychologie 66:91-115.

Kalter, Frank and Hanno Kruse. 2014. "Ethnic diversity, homophily, and network cohesion in European classrooms." Pp. 187-207 in Social Cohesion and Immigration in Europe and North America: Mechanisms, Conditions, and Causality, eds. Ruud Koopmans, Bram Lancee, and Merlin Schaeffer. London: Routledge.

Knecht, Andrea, Tom A. B. Snijders, Chris Baerveldt, Christian E. G. Steglich, and Werner Raub. 2010. "Friendship and delinquency: Selection and influence processes in early adolescence." Social Development 19:494-514.

Koskinen, Johan H. and Tom A. B. Snijders. 2020. Multilevel Longitudinal Analysis of Social Networks. In preparation. Groningen/Melbourne.

Kossinets, Gregory and Duncan J. Watts. 2009. "Origins of homophily in an evolving social network." American Journal of Sociology 115:405-450.

Kruse, Hanno and Konstanze Jacob. 2016. Children of Immigrants Longitudinal Survey in Four European Countries. Sociometric Fieldwork Report. Technical report, Mannheim University, Mannheim. Wave 1-2010/2011, v1.2.0.

Kruse, Hanno, Markus Weißmann, and Konstanze Jacob. 2016. Children of Immigrants Longitudinal Survey in Four European Countries. Sociometric Fieldwork Report. Technical report, Mannheim University, Mannheim. Wave 2-2011/2012, v2.3.0.

Lospinoso, Joshua A. and Tom A. B. Snijders. 2019. "Goodness of fit for stochastic actor-oriented models." Methodological Innovations 12:2059799119884282.

Macy, Michael W. and Robb Willer. 2002. From factors to actors: Computational sociology and agent-based modeling." Annual Review of Sociology 28:143-166.

Manzo, Gianluca. 2010. "Analytical sociology and its critics." European Journal of Sociology/ Archives européennes de sociologie 51:129-170.

McPherson, Miller, Lynn Smith-Lovin, and James M. Cook. 2001. "Birds of a feather: Homophily in social networks." Annual Review of Sociology 27:415-444.

Moody, James. 2001. "Race, school integration, and friendship segregation in America." American Journal of Sociology 107:679-716.

Moody, James and Douglas R. White 2003."Structural cohesion and embeddedness: A hierarchical concept of social groups." American Sociological Review 68:103-127.

Mouw, Ted and Barbara Entwisle. 2006. "Residential segregation and interracial friendship in schools." American Journal of Sociology 112:394-441.

Putnam, Robert D. 2007. "E pluribus unum: Diversity and community in the twenty-first century." Scandinavian Political Studies 30:137-174.

Raub, Werner. 1984. Rationale Akteure, institutionelle Regelungen und Interdependenzen. Untersuchungen zu einer erklärenden Soziologie auf strukturell-individualistischer Grundlage. Frankfurt am Main: Lang.

Raub, Werner, Vincent Buskens, and Marcel A. L. M. van Assen. 2011. "Micro-macro links and microfoundations in sociology." Journal of Mathematical Sociology 35:1-25.

Ripley, Ruth M., Tom A. B. Snijders, Zsófia Bóda, András Vörös, and Paulina Preciado. 2020. Manual for Siena Version 4.0. Technical report, Oxford: University of Oxford, Department of Statistics; Nuffield College. 
Schaeffer, Merlin. 2013. Ethnic Diversity, Public Goods Provision and Social Cohesion: Lessons from an Inconclusive Literature. Discussion Papers, Research Unit: Migration, Integration, Transnationalization SP VI 2013-103, WZB Berlin Social Science Center.

Scott, J. and P. J. Carrington (eds.). 2011. The SAGE Handbook of Social Network Analysis. London: Sage.

Simmel, Georg. 1917 [1950]. "Individual and society." Pp. 58-86 in The Sociology of Georg Simmel, ed. K. Wolff. New York: The Free Press.

Snijders, Tom A. B. 2001. "The statistical evaluation of social network dynamics." Sociological Methodology 31:361-395.

Snijders, Tom A. B. 2016. "The multiple flavours of multilevel issues for networks." Pp. 15-46 in Multilevel Network Analysis for the Social Sciences; Theory, Methods and Applications, eds. Emmanuel Lazega and Tom A. B. Snijders. Cham: Springer.

Snijders, Tom A. B. 2017. "Stochastic actor-oriented models for network dynamics." Annual Review of Statistics and Its Application 4:343-363.

Snijders, Tom A. B. and Chris Baerveldt. 2003. "A multilevel network study of the effects of delinquent behavior on friendship evolution." Journal of Mathematical Sociology 27:123-151.

Snijders, Tom A. B. and Roel J. Bosker. 2012. Multilevel Analysis: An Introduction to Basic and Advanced Multilevel Modeling (2nd ed.). London: Sage.

Snijders, Tom A. B. and Christian Steglich 2015. "Representing micro-macro linkages by actorbased dynamic network models.” Sociological Methods \& Research 44:222-271.

Snijders, Tom A. B., Gerhard G. van de Bunt, and Christian E. G. Steglich 2010. "Introduction to actor-based models for network dynamics." Social Networks 32:44-60.

Steglich, Christian E. G., Tom A. B. Snijders, and Michael Pearson. 2010. "Dynamic networks and behavior: Separating selection from influence.” Sociological Methodology 40:329-393.

Stokman, Frans N. and Manuela Vieth. 2004. "Was verbindet uns wann mit wem? Inhalt und Struktur in der Analyse sozialer Netzwerke." Kölner Zeitschrift für Soziologie und Sozialpsychologie, Sonderheft 44:274-302.

van der Meer, Tom and Jochem Tolsma. 2014. "Ethnic diversity and its effects on social cohesion." Annual Review of Sociology 40:459-478.

Wimmer, Andreas and Kevin Lewis. 2010. "Beyond and below racial homophily: ERG models of a friendship network documented on Facebook." American Journal of Sociology 116:583-642.

Windzio, Michael and Matthias Wingens. 2014. "Religion, friendship networks and home visits of immigrant and native children." Acta Sociologica 57:59-75. 
